\title{
Constraining astrophysical reaction rates: using the storage rings at FAIR/GSI
}

\author{
Christoph Langer ${ }^{1, \star}$, Jan Glorius ${ }^{2}$, Zuzana Slavkovská ${ }^{1}$, Sergey Litvinov², Yuri A. Litvinov², and \\ René Reifarth ${ }^{1}$ \\ ${ }^{1}$ Goethe University Frankfurt, Max-von-Laue Strasse 1, Frankfurt a. M., Germany \\ ${ }^{2}$ GSI Darmstadt, Planckstrasse 1, 64291 Darmstadt, Germany
}

\begin{abstract}
Ion optical calculations for a storage ring at the present GSI facility for direct proton-induced reactions relevant for different astrophysical processes are presented. As an example case, the ${ }^{59} \mathrm{Cu}(p, \gamma)$ and ${ }^{59} \mathrm{Cu}(p, \alpha)$ reactions are shown. The branching of these two reactions is important in X-ray burst scenarios, since it determines the breakout out of the major ${ }^{56} \mathrm{Ni}$ waiting point.
\end{abstract}

\section{Introduction}

One of the biggest challenges in Nuclear Astrophysics is the extrapolation of charged-particle induced cross sections, typically measured at high energies, to the astrophysically relevant low energies. This process relies heavily on predictions from theory and often results in large uncertainties and even conflicting statements. As such, direct measurements at the low stellar energies would be ideal.

However, measurements of charged-particle induced reactions, like $(p, \gamma),(\alpha, \gamma),(\alpha, p)$, typically suffer from extremely low cross sections at the interesting stellar energies. One way to overcome this problem experimentally is to increase the number of beam particles. This is of course limited by each facility and especially for radioactive ions, it is mostly impossible.

If radioactive ions are involved in the reaction, almost always experiments in inverse kinematics need to be performed. However, the production rates of radioactive ions at currently operational radioactive beam facilities are often still too low to perform direct experiments in inverse kinematics with the standard geometry of a fixed target and the beam impinging on it once (single pass experiment). This approach has its own advantages; however, the unreacted beam after the target is typically lost for further reactions.

In this respect, astrophysical reaction measurements employing storage rings (multi pass) enable unprecedented studies of reactions, which are not accessible so far. High luminosities on the order of $10^{25} \mathrm{~cm}^{-2} \mathrm{~s}^{-1}$ can be achieved at the relevant astrophysical energies (see Fig. 1, in which different Gamow window ranges for some astrophysical processes are shown). This leads to acceptable rates even for relatively low cross sections. These luminosities are achieved by revolving the unreacted beam at a high frequency in a storage ring while constantly readjusting the momentum spread of the beam (e.g. induced while traversing the target) by using an electron cooler. This device efficiently cools the beam to a given energy spread (which can be very well fine-tuned).

\footnotetext{
^e-mail: langer@iap.uni-frankfurt.de
} 


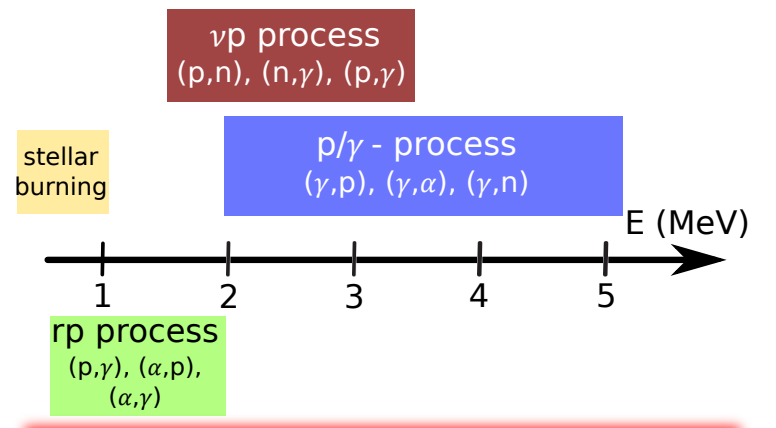

$v p$ process

$(p, n),(n, \gamma),(p, \gamma)$

CRYRING @ ESR (min. magnetic rigidity $0.054 \mathrm{Tm}$ )

ESR (min. magnetic rigidity $0.5 \mathrm{Tm}$ )
Figure 1. Typical Gamow window energy ranges for $(p, \gamma)$ reactions for different astrophysical processes. Other reactions involved in the particular process are also shown. The expected experimental coverage of the future CRYRING@ESR [7] and the existing ESR storage ring at GSI [1] can be seen.

Following the reaction in a gas-jet target, the product has a different magnetic rigidity than the beam according to $B \rho \sim A / Z$. As a consequence, the trajectories in a dipole are differing. A standard position-sensitive particle detector, like a Si detector (DSSSD), is then inserted to detect the products in the dipole without intercepting the circulating unreacted beam. Fig. 2 shows the kinematical relationship between the laboratory energy (typically denoted in $\mathrm{MeV} / \mathrm{A}$ ) and the center-of-mass system, corresponding to the Gamow window. As can be seen: $\alpha$-induced reactions require much lower beam energies, which inherently complicates the experiment.

The success of this method using the existing ESR storage ring at GSI [1] has been shown in [2] and in a recent experiment employing the ${ }^{124} \mathrm{Xe}(p, \gamma)$ reaction [3]. Both reactions belong to the astrophysical $\gamma$-process and the measurements allow to constrain input parameters for Hauser-Feshbach rate calculations.

In the following chapter, ion-optics calculations are presented for a typical reaction important for Nuclear Astrophysics showing possibilities and current limitations.

\section{Kinematics and ion-optics calculations}

The rapid proton-capture (rp) process drives the energy generation of type I X-ray bursts [4]. An important branching point is identified at the radioactive ${ }^{59} \mathrm{Cu}$ isotope. It has been shown recently, that the $(p, \gamma)-(p, \alpha)$ branching on ${ }^{59} \mathrm{Cu}$ determines the breakout out of the important ${ }^{56} \mathrm{Ni}$ waiting point $[5,6]$, see left part of Fig. 3. None of the reactions involved (i.e. ${ }^{59} \mathrm{Cu}(p, \gamma)$ and $\left.{ }^{59} \mathrm{Cu}(p, \alpha)\right)$ have been measured so far, especially not in the astrophysically relevant energy region.

This is an interesting candidate reaction to be studied using the storage rings at FAIR/GSI. The isotope ${ }^{59} \mathrm{Cu}$ can be sufficiently produced using the fragmentation method at GSI. Its half-life is long enough to be stored easily for some time in the ring for preparation of the beam and carrying out the measurement. A dense liquid hydrogen target $\left(\approx 10^{14}\right.$ particles $\left./ \mathrm{cm}^{2}\right)$ is used for the reaction surrounded by X-ray detectors for normalization of the cross section [2]. A standard double-sided silicon strip detector is placed into the ultra high vacuum of the storage ring in the dipole for detection of the reaction residuals, which move on a different trajectory than the unreacted beam. In a recent experiment, the feasibility of this method has been shown successfully [3].

The maximum deflection angle of the ${ }^{60} \mathrm{Zn}$ product after the ${ }^{59} \mathrm{Cu}(p, \gamma)$ reaction is 0.1 degrees, as can be seen in the right part of Fig. 3. In the case of ${ }^{56} \mathrm{Ni}$ following the ${ }^{59} \mathrm{Cu}(p, \alpha)$ reaction, the maximum angle is 3.7 degrees. Typically, in the storage rings at GSI, the particles drift behind the target for several meters before they enter into the first quadrupoles. The large difference in 


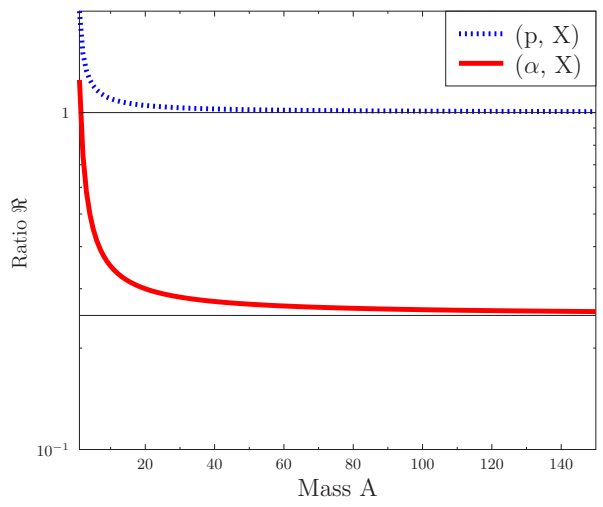

Figure 2. The kinematical ratio $\mathfrak{R}$ defined here as $E_{l a b}^{*} / E_{C M}$, in which $E_{l a b}^{*}$ is given in the typical units of $M e V / A$ used for the beam energy. If $e . g$. a $(p, \gamma)$ reaction with $A=59$ needs to be studied in inverse kinematics and the Gamow window centers at $4 \mathrm{MeV}$, a beam energy of $\mathfrak{R}(A=59) \times 4=4.1 \mathrm{MeV} / \mathrm{A}$ is required. On the other hand, for the same mass but an $(\alpha, \gamma)$ reaction, a beam energy of $1.3 \mathrm{MeV} / \mathrm{A}$ is required (since $\mathfrak{R} \approx 0.3$ ), which is experimentally much more challenging.

the laboratory scattering angle between the two reactions results in significant differences in the $x-y$ particle distribution just before the first quadrupoles, as can be seen in the left part of Fig. 4. Also indicated is the beam pipe aperture, which significantly cuts into the acceptance actually required for the $(p, \alpha)$ reaction case. It is subsequently clear, that a measurement of a $(p, \alpha)$ reaction is much more challenging and requires a different approach compared to the $(p, \gamma)$ case. For an induced $(p, \gamma)$ reaction, the heavy residual nucleus moves almost on the same trajectory as the unreacted beam before the dipole. Because of the different $A / Z$-ratio, the magnetic rigidity is different for the unreacted beam and the residual. Consequently, the trajectories inside and after the dipole are differing and this allows to disentangle both: whereas the unreacted beam is recycled in the ring, the residual is detected with almost $100 \%$ detection efficiency in the DSSSD.

As can be concluded from Fig. $4,{ }^{59} \mathrm{Cu}(p, \gamma)$ can be measured without any losses due to acceptance. A DSSSD with standard size of $(5 \times 5) \mathrm{cm}^{2}$ covers the complete distribution of the $(p, \gamma)$ reaction residuals in the dipole at a distance to the unreacted revolving beam of $\approx 8 \mathrm{~cm}$. The ${ }^{59} \mathrm{Cu}(p, \alpha)$ residuals will be cut almost completely by the acceptance of the ESR and, thus, are not measurable using
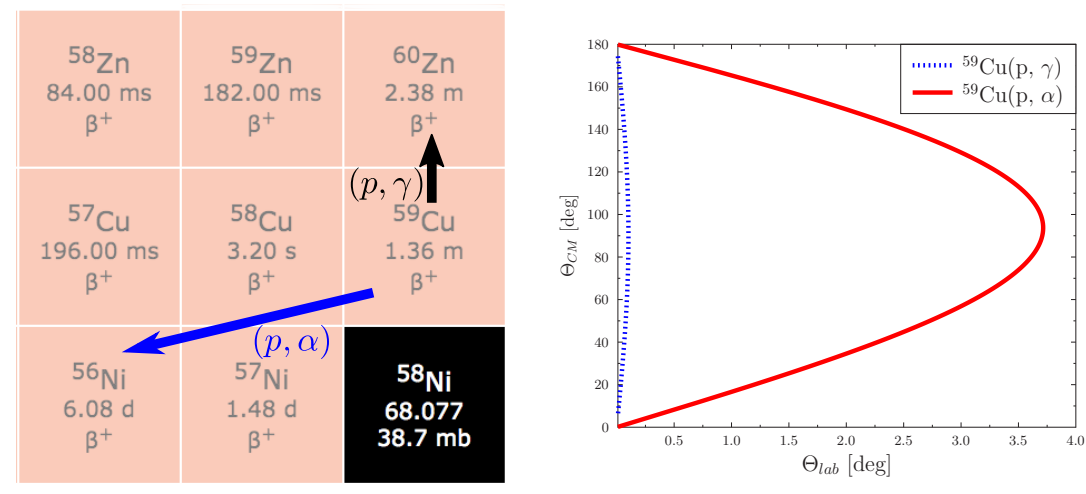

Figure 3. Left: Part of the rp process around the important ${ }^{56} \mathrm{Ni}$ waiting point. The ${ }^{59} \mathrm{Cu}(p, \gamma)$ reaction leads to ${ }^{60} \mathrm{Zn}$, and, as such, successfully breaking out of the ${ }^{56} \mathrm{Ni}$ waiting point. The competing reaction ${ }^{59} \mathrm{Cu}(p, \alpha)$, instead feeds back into ${ }^{56} \mathrm{Ni}$. Right: Transformation of the center-of-mass scattering angle of the heavy residuals into the laboratory azimuthal angle $\Theta_{l a b}$ for the two example reactions considered here. The CM energy of both reactions is set to $4 \mathrm{MeV}$ to be still measurable in the ESR (see Fig. 2). 

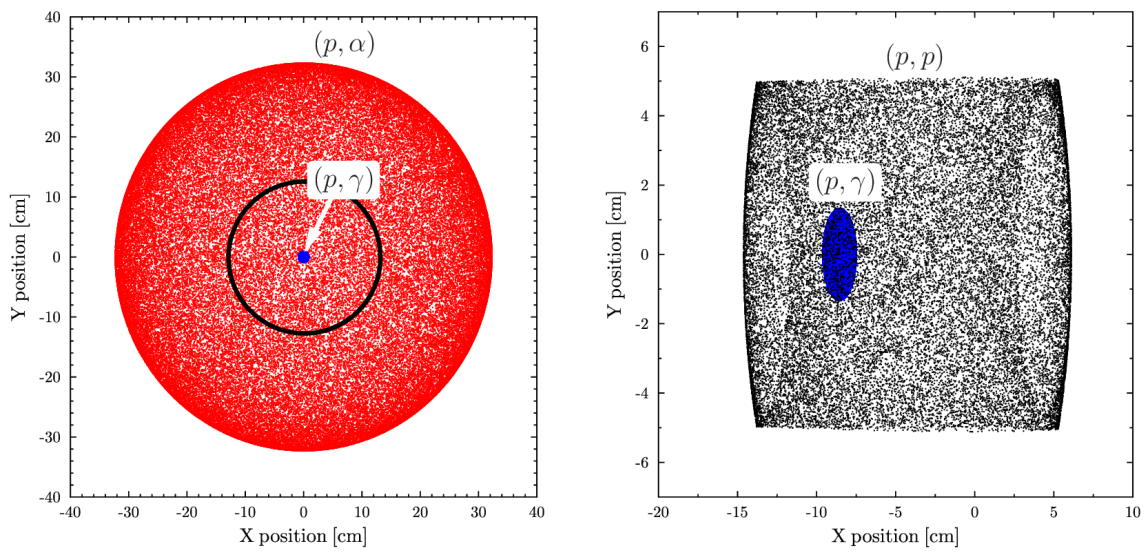

Figure 4. Left: The $\mathrm{x}-\mathrm{y}$ distribution of heavy residuals following the ${ }^{59} \mathrm{Cu}(\mathrm{p}, \alpha)$ and ${ }^{59} \mathrm{Cu}(\mathrm{p}, \gamma)$ reaction before entering into the first quadrupole in the ESR after the target (drift path is roughly 5 meters). MOCADI has been used to simulate the beam transport [8]. The black circle indicates the typical ESR beam line aperture. Right: The $\mathrm{x}$-y distribution in the dipole (at 53.3 degrees, which is the position used in [3]) of the ${ }^{59} \mathrm{Cu}(p, \gamma)$ residuals. They can be sufficiently measured with a $(5 \times 5) \mathrm{cm}^{2}$ DSSSD detector. In black, also the elastic scattering contribution from ${ }^{59} \mathrm{Cu}(\mathrm{p}, \mathrm{p})$ is shown. The MOCADI simulations include realistic beam apertures; for the reaction, no internal decay patterns of the single residuals are incorporated.

this technique and measurement position. According to Rutherford scattering, at low center-of-mass energies the elastic scattering contribution is expected to be high. The distribution of the $(p, p)$ events can also be seen in the right part of Fig. 4. However, as has been shown before, depending on the cross section of the ${ }^{59} \mathrm{Cu}(p, \gamma)$ reaction, it should be possible to disentangle both distributions [2, 3]. Another idea could be to block the elastic scattering contribution already before entering into the dipole, since its spread is much wider than in the $(p, \gamma)$ case.

\section{References}

[1] B. Franzke, Nuclear Instruments and Methods in Physics Research Section B: Beam Interactions with Materials and Atoms 24, 18 (1987)

[2] B. Mei, T. Aumann, S. Bishop, K. Blaum, K. Boretzky, F. Bosch, C. Brandau, H. Bräuning, T. Davinson, I. Dillmann et al., Phys. Rev. C 92, 035803 (2015)

[3] Experiment E108b at GSI in 2016

[4] H. Schatz, A. Aprahamian, J. Görres, M. Wiescher, T. Rauscher, J. Rembges, F.K. Thielemann, B. Pfeiffer, P. Möller, K.L. Kratz et al., Physics Reports 294, 167 (1998)

[5] R.H. Cyburt, A.M. Amthor, A. Heger, E. Johnson, L. Keek, Z. Meisel, H. Schatz, K. Smith, The Astrophysical Journal 830, 55 (2016)

[6] C. Langer, N. Klapper, C. Köppchen, S. Dababneh, R. Reifarth, submitted to Jordanian Journal of Physics (2017)

[7] M. Lestinsky, V. Andrianov, B. Aurand, V. Bagnoud, D. Bernhardt, H. Beyer, S. Bishop, K. Blaum, A. Bleile, A. Borovik et al., The European Physical Journal Special Topics 225, 797 (2016)

[8] H. Weick et al., https://web-docs.gsi.de/ weick/mocadi/index.html, 2017 